Penultimate draft. For published version see:

https://www.tandfonline.com/doi/full/10.1080/00048402.2021.1908378

\title{
SILENCE PERCEPTION AND SPATIAL CONTENT
}

Błażej Skrzypulec

It seems plausible that visual experiences of darkness have perceptual, phenomenal content which clearly differentiates them from absences of visual experiences. I argue, relying on psychological results concerning auditory attention, that the analogous claim is true about auditory experiences of silence. More specifically, I propose that experiences of silence present empty spatial directions like 'right' or 'left', and so have egocentric spatial content. Furthermore, I claim that such content is genuinely auditory and phenomenal in the sense that one can, in principle, recognize that she is experiencing silence. This position is far from obvious as the majority of theories concerning silence perception do not ascribe perceptual, phenomenal content to experiences of silence.

Keywords: silence; audition; absence perception; spatial perception; content

\section{Introduction}

We do not see anything behind our head. We also do not see anything in a completely dark room. Though both these situations can be described as cases of 'not seeing,' there is a significant difference between them. In the first case, we do not see in the sense of a lack of visual experience, but in the second, we have a visual experience that presents darkness filling the whole visual field [Sorensen 2004; Wright 2012]. If one accepts the representationalist 
approach to perceptual states, the difference between absence of a visual experience and the perception of total darkness can be described in terms of representational content, that is, as a difference between having and not having some accuracy conditions [Siegel 2010]. A visual experience of darkness has some content; let's say there is darkness in every direction, but if there is no visual experience, then there is no representational content at all. Furthermore, the content of the experience of darkness is such that, in principle, a subject can introspectively recognize that she is experiencing darkness. For the purpose of the paper, I call such content 'phenomenal content'. ${ }^{1}$ Finally, the content of darkness experiences is plausibly a perceptual content. A visual experience of darkness has its content due to the function of perceptual mechanisms, and not due to some higher-order processes which may result in a belief that it is dark.

Overall, it seems plausible that visual experiences of darkness have perceptual, phenomenal content that differentiates them from an absence of visual experience. This paper argues that the analogous claim is true about auditory experiences of silence. More specifically, I claim that experiences of silence present empty spatial directions like 'right' or 'left,' and so have egocentric spatial content. I argue for this thesis from the perspective of a representationalist account of perception, according to which perceptual experiences are representations possessing content that determines their accuracy conditions. While I believe that my arguments could be also expressed in terms closer to direct realist accounts, for instance, by claiming that an experience of silence has an empty direction as its constituent, the justification of this claim goes beyond the scope of the paper.

\footnotetext{
${ }^{1} \mathrm{I}$ do not postulate a stronger thesis that an ability for introspective recognition is necessary for content to be phenomenal. I merely postulate that such an ability is present in the case of experiences of darkness, and later argue that the same is true of experiences of silence.
} 
The proposed account is far from obvious, as there is no agreement regarding whether experiences of silence can be distinguished from absences of auditory experience by postulating perceptual, phenomenal content. In particular, experiences of silence do not seem to be associated with any positive phenomenal quality, such as darkness, which differentiates them from absences of auditory experience. In fact, the major theories concerning silence perception do not treat experiences of silence as having perceptual, phenomenal content [O'Shaughnessy 2000; Sorensen 2009]. One theory that may be interpreted with some plausibility as attributing such content to experiences of silence has been proposed independently by Soteriou [2011] and Philips [2013]. According to this theory, when hearing silence, we experience an empty temporal region. If such claim is expressed in terms of temporal content, then it can be proposed that experiences of silence have content, such as there is an empty temporal region from $t_{x}$ to $t_{y}$. It is likely that such content is phenomenal and perceptual, as there seems to be a certain conscious awareness related to the experience of temporal regions due to the function of perceptual mechanisms [Soteriou 2011: 199-201]. Nevertheless, neither Soteriou nor Phillips postulates that experiences of silence have any spatial content in addition to temporal content.

I start by presenting my theory of silence perception according to which experiences of silence have egocentric, directional spatial content that is perceptual and phenomenal (Section 2). The argument for this thesis, presented in Section 3, takes the form of an inference to the best explanation. I rely on empirical results obtained in auditory priming studies and claim that they make my thesis regarding the content of experiences of silence more plausible than the major alternative views. This claim is laid out in four major steps. First, I argue that priming studies strongly suggest the presence of egocentric, directional content in the case of experiences of silence (Section 3.1). Second, I provide reasons for believing that such content is not merely derivative. In particular, its occurrence does not 
depend on the presence of an earlier perception of an auditory stimulus with spatial characteristics (Section 3.2). Third, I argue that the results of priming studies make it more plausible that the considered spatial content is auditory than that it is not perceptual at all, is connected to different modality than audition such as vision, or is amodal (Section 3.3). Fourth, by relying on the way in which endogenous attention functions in priming studies and general views regarding relations between attention and consciousness, I claim that the spatial content of experiences of silence is likely to be phenomenal in a perceptual, and not merely a cognitive way (Section 3.4). Finally, in Section 4, I postulate that my spatial theory has certain advantages over temporal theory, and defend my theory against Meadows' [2019] arguments that experiences of silence do not have spatial content.

\section{Space, Audition, and Silence Perception}

My main focus is pure experiences of silence in which one literally does not hear any sounds. In practice, such experiences are rare, as one is usually able to perceive some background sounds, and a lack of external auditory stimulation often leads to interoceptive auditory experiences concerning, for instance, heartbeats. Nevertheless, experiences of pure silence seem to be possible for human audition, and checking whether a theory of silence perception can account for pure such experiences constitutes a useful test of its validity [Phillips 2013: 348]. Furthermore, I also apply my spatial conception of experiences of silence to partial experiences of silence. In such experiences, which are probably more common than those of pure silence, one hears that there are sounds in some parts of one's surroundings, but other parts are silent. For instance, one may experience a sound on the left but no sounds on the right. 
I argue that audition represents an empty auditory space in experiences of both pure and partial silence. In doing so, I assume that standard auditory experiences have spatial content, as they represent auditory entities, usually understood as sounds or their sources, as located in some direction from the perceiver. While the auditory ability to represent space has been a matter of philosophical controversy [O‘Callaghan 2008; O’Shaughnessy 2009; Nudds 2009; Young 2017], I believe that there are good reasons to accept that human audition can represent at least the directions of auditory entities. First, the phenomenology of ordinary auditory experiences strongly suggests that we can differentiate between hearing something on our right from hearing something on our left. Second, there are specialized auditory physiological mechanisms which allow us to detect the direction of auditory stimuli by comparing the time at which sound waves stimulate receptors in each ear (see Moore [1991] for review). Third, experimental data from psychology show that one can focus auditory attention toward the left or right and more efficiently process stimuli presented from the chosen direction (more on this in Section 3).

It should be noted that postulating auditory spatial content concerning the directional properties of auditory entities does not entail accepting that auditory spatial content is analogous to the spatial content of visual experiences in which objects are presented as positioned in a bounded, topologically connected field [Mac Cumhaill 2015; Richardson 2010]. Further, I argue that the spatial content in case of experiences of silence is similar to the spatial content of experiences presenting auditory entities, as it also concerns spatial directions. More precisely, I claim that experiences of silence have spatial content that is empty, egocentric, and directional. In the case of experiences of pure silence it can be described as there is an empty direction 'left' and an empty direction 'right', while partial experiences of silence have content which specifies that only some directions are empty, like there is an empty direction 'left' and a sound $X$ in direction 'right.' Such spatial content is 
directional, as it concerns directions in space; it is egocentric, as the directions are determined relative to the perceiving subjects; and it is empty, as it ascribes the characteristic of emptiness to experienced directions. In consequence, my account entails that the content of auditory experiences cannot be fully accounted for in terms of sounds and sound sources possibly having spatial properties, as there are at least some auditory experiences, such as experiences of silence, whose content involves a different type of entities: spatial directions. Subsequently, I argue only for a thesis that what is spatially presented in experiences of silence are two directions: left and right. However, nothing in my reasoning is incompatible with possible stronger theses that would claim that more directions are auditorily represented.

There is also a type of experience of silence I do not attempt to analyze in terms of spatial, directional content. These are cases of silence perception in which one experiences periods of silence between sounds or within the structure of complex auditory stimuli. For instance, listening to a musical piece may involve experiencing silent gaps between sounds, and such gaps may be perceived as parts of the overall complex stimuli contributing to its temporal structure. I believe that such cases of silence perception can be adequately grasped by a 'contrast theory,' in which the perception of silence is analyzed in terms of perceiving the endings and beginnings of sounds (see Meadows [2020]; Phillips [2013]; Soteriou [2011] for a discussion). From this perspective, silence perception seems to be analogous to visual hole perception with the difference that auditory holes are temporal rather than spatial. Alternatively, some experiences of auditory temporal gaps can be explained by theories postulating that the perception of absences arises from violations of perceptual expectations [Cavedon-Taylor 2017; Farennikova 2013; Martin and Dokic 2013]. For example, one may experience a pattern of sounds such that the next sound is presented half a second after the previous one. When the series end, a person may have an experience of silence with content determined by the violated expectation concerning the continuation of the series. 
Nevertheless, such theories are not well-suited for explaining experiences of pure silence in which no beginnings or endings of sounds are represented and no expectations are formed based on previously heard sounds.

\section{Experiences of Silence and Spatial Content}

\subsection{Spatial Attention and Directional Content}

It is well-established that visual attention is both space- and object-based (see Scholl [2001] for review). This means that one may attentively choose a certain object and process it more efficiently or may focus attention on a spatial region in the visual field such that whatever appears in this region is processed in a privileged way.

Space-based visual attention can be exogenous or endogenous. In the exogenous case, a priming stimulus drags attention to a certain region, and new elements appearing in this region are processed attentively even after the priming stimulus disappears. Endogenous attention is not attributed to a region of space in virtue of an influence of an external stimulus, but is voluntarily applied by a subject relying, for instance, on beliefs concerning the likely location of the relevant stimulus. Both in the case of applying exogenous and endogenous visual space-based attention, there may be a period in which attention is focused on a place in which no relevant stimulus is present. This period can occur before presentation of a stimulus (endogenous attention) or between a priming stimulus and a later stimulus (exogenous attention).

The presence of space-based visual attention suggests that vision can represent spatial regions. Major theories of visual attention treat attention as a selective mechanism that chooses elements to allow for their more elaborate processing (see the coherence theory of 
attention [Rensink 2000]; and feature integration theory [Treisman 1998]). It seems plausible that if some element is visually, attentionally processed, then it figures in the representational content of a given experience. It is so because attended elements are those to which we have privileged phenomenal access, and which are perceptually processed in a detailed way [Carrasco and Barbot 2019; Prinz 2000]. For instance, using a spatial example, if a circular region is visually attended, then a visual experience presents that there is a circular region or that this region is circular (depending on whether perceptual content is interpreted as general or particular, see Schellenberg [2016]). The close relationship between attention and consciousness makes it plausible that the attentionally processed elements figure in the experiential content of conscious, perceptual experiences, and do not merely constitute some subpersonal content. In fact, the only cases in which attentional processing occurs without perceptual awareness of the stimuli are specific situations in which a very brief stimulus is presented in the attended region, or in which two attended stimuli are arranged such that one suppresses the awareness of the another (see Boxtel et al. [2010] for review). Of course, the experiential content may vary depending on the amount of attributed attentional resources. For instance, with focused attention, a circular region may be experienced as having a specific shape, while with diffused attention, it is experienced merely as a convex region without further specification.

I believe that the analogous story is true in case of auditory perception and that it can be generalized to account for experiences of pure and partial silence. More specifically, I claim that the way in which auditory attention works suggests that, even if no sounds are heard, audition presents, due to attentional processing, empty spatial directions. While the early results were inconclusive (see Spence and Driver [1996] for review), there is now a widespread conviction that phenomena related to visual spatial attention have counterparts in the auditory modality. In a common experimental design [Mondor and Bryden 1992; Sach et 
al. 2000; Tata and Ward 2005], a participant hears an auditory cue on the left or on the right and then, after a period of silence, a target sound is presented at the location of the earlier cue. It has been observed that cuing makes processing the subsequent sound, positioned in the direction of cue, more efficient, as the reaction time needed for recognizing the stimulus' properties is shorter and responses are more accurate.

Such results suggest that auditory attention can select an empty spatial direction such as 'left' or 'right.' It is so because between a presentation of a cue and a presentation of a target sound there is a period of silence during which attention is still focused in one of the directions. As a result of the attentional focus, subsequent stimuli positioned in the direction attended are processed in a privileged way. Because attention selects an empty direction, it is plausible to accept that this direction figures in the representational content of a given auditory experience. In consequence, psychological results support a thesis that there are experiences of silence which have empty, directional, egocentric content. What is auditorily presented between a cuing sound and a target sound is that there is an egocentrically determined spatial direction, such as 'left,' and that this direction is empty.

\subsection{Derivativity Problem}

However, there are two reasons that prevent us from generalizing the interpretation above and applying it to experiences of pure silence. First, in the described experimental design, auditory attention is exogenous, as attention is dragged toward one of the directions by the cuing stimulus. Nevertheless, in the case of experiences of pure silence, the focus of attention must be directed endogenously, as by definition there are no auditory stimuli which would attract attention in such experiences. Second, it may be the case that in experiences of silence occurring during cuing studies the spatial content is merely derivative. More 
specifically, one may claim that an empty spatial direction can be auditorily represented only if there was a sound represented as positioned in this direction a moment earlier. According to such a position, the auditory system can retain information about the spatial location of a sound for some time and represent this location even when a sound is no longer present. In this way, auditory experiences of empty space are always dependent on previous experiences of sounds possessing spatial properties. Of course, in an experience of pure silence, such dependence cannot be obtained, and so they may not have any spatial content.

Nevertheless, it is believed that the cuing paradigm can also engage endogenous attention in certain circumstances [Mayer et al. 2006]. In particular, when the proportion of sounds coming from left and right is not equal, for instance, $80 \%$ of sounds presented from the right and $20 \%$ from the left, participants tend to endogenously focus attention toward the direction from which sounds are presented more frequently, and due to allocation of attentional resources, these sounds are processed more efficiently. When such a procedure is applied, there are moments between presentations of sounds in which endogenous attention is focused toward an empty, egocentric direction such as 'left' or 'right.' In consequence, not only by using exogenous attention but also by using endogenous attention one can obtain an experience of silence that presents an empty spatial direction. However, the spatial content of these experiences can still be interpreted as derivative as there are frequent sounds positioned in the direction to which endogenous attention is focused.

I believe that the problem concerning the possible derivative nature of empty auditory spatial content can be overcome, as there are also studies in which endogenous attention is spatially directed without prior auditory spatial cuing. In some such studies, participants are presented with a visual symbolic cue (see experiments 2 and 5 in Sach et al. [2000]; Schröger and Eimer [1997]; experiments 1-3 and 6 in Spence and Driver [1996]; Voisin et al. [2006]). For instance, participants may be shown an arrow that points right. Relying on this cue, they 
endogenously direct auditory attention to the right, and if a sound is subsequently presented in this direction, it is processed more efficiently. Alternatively, the information concerning the direction is not given in the form spatially positioned auditory cue but by providing propositional data (see experiments 4 and 7 in Spence and Driver [1996]; Teder-Sälejärvi and Hillyard [1998]; Wu et al. [2007]). For instance, before beginning an experiment, a participant is instructed, verbally or in writing, to direct auditory attention in a particular direction.

The initial period of the tasks described above is a close empirical approximation of an experience of pure silence, as after receiving a visual cue or being given an instruction there is a period in which a person does not hear any sounds. In particular, irrelevant sounds are excluded in auditory attention experiments by presenting stimuli through headphones or in an isolated room. The results of the experiments described above show that participants are able to endogenously direct auditory attention towards an egocentric direction such as 'right' or 'left' even without auditory spatial cuing. Thus, this ability does not depend on a previous presentation of a sound positioned in the direction considered. In consequence, it seems that even in the case of an experience of pure silence, auditory attention can select an empty spatial direction. This makes it plausible to assume that experiences of pure silence have empty, egocentric, directional content. This conclusion is applicable both to experiences of pure silence and to experiences of partial silence in which only one empty direction is presented.

However, two doubts can arise concerning the above interpretation. First, while the direction of attention in the experiments considered is not cued by a spatially directed sound, it is preceded by a visual or propositional stimulus. If this is the case, then it is possible that empty directional content is still derivative, as it depends on certain input providing spatial information. Second, I assume that attentional focus is a selective mechanism, such that attention focuses on elements that are already represented. In the context of silence 
perception, this means that empty spatial directions are already represented even before attention is focused. While such an assumption may be plausible in the case of visual spatial attention, one may doubt whether the same can hold for the auditory modality. Perhaps it is not the case that attention is focused toward a direction that is already represented, but rather, that attentional focus initiates an auditory spatial representation.

The first worry can be understood in either a stronger or weaker sense. The stronger interpretation poses a threat to my position but is implausible, while the weaker interpretation is likely true, but is not problematic for my thesis regarding the spatial perception of silence. According to the stronger interpretation, to pay attention to direction $X$, visual or propositional stimulus carrying information about direction $X$ is necessary. For instance, in virtue of presenting the word 'right', a person can direct attention to the right but not to the left, as the relevant spatial information is not provided by this stimulus. However, such an interpretation is not plausible, as it seems entirely possible that a person may decide that she will focus her attention to the left upon seeing the word 'right', or that a person may focus attention to the left or right relying not on any external stimuli but in virtue of a belief or an expectation. The second, weaker interpretation states that visual or propositional input has a causal role, such that it evokes spatial beliefs or expectations necessary for focusing endogenous auditory attention to the left or right. For instance, seeing the word 'right' may cause an expectation that an interesting stimulus will occur to the right. However, such beliefs and expectations can also arise independently of external stimulation, or even in opposition to it, as in the case of focusing attention to the left when seeing the word 'right'. While this interpretation is plausible, it does not entail that spatial content in case of experiences of silence is derivative in any relevant sense. It is a common feature of endogenous spatial attention-visual or auditory - that its allocation relies on some state having spatial content, such as a belief that one should focus to the right. In consequence, while the spatial content of experiences of 
silence may be 'derivative' in this sense, it is no more so than analogous visual spatial content.

According to the second worry, it is not the case that an experience of pure silence has spatial content before attention is focused to the right or left. It should be noted that even if such a hypothesis is true, it is not inconsistent with a thesis that experiences of silence has directional spatial content. It would only restrict the scope of the thesis to situations in which endogenous attention is employed when perceiving silence. However, I believe that there are data which allow me to defend a stronger thesis that directional spatial content is present in experiences of pure silence even without attentional focus. As stated above, it has been observed that when auditory attention is focused toward one direction, auditory stimuli presented in this direction are processed more efficiently. Furthermore, an opposite effect has also been reported: if auditory attention is focused towards one direction, let's say 'right', then an auditory stimulus presented on the left is processed less efficiently than in a case in which attention is not directionally focused at all (see Schröger and Eimer [1997]; experiment 1 in Spence and Driver [1996]). After focusing in one direction (in this example, the right), attentional resources initially allocated to the left direction are directed to the right, leading to a decrease in the processing of left-located stimuli. This suggests that before directional focus, attention is distributed toward both directions, 'left' and 'right,' and so both directions are represented at least in some rudimentary form. If auditory spatial directions were not represented at all before attentional focus, then prior to attention being focused, the processing of auditory stimuli in both directions should be at least as poor as in the case of processing left-located stimuli when attention is directed towards right. However, this is not what experimental results reveal. In consequence, it is plausible to state that even before attentional focus both auditory egocentric directions are represented by using attentional resources. However, their pre-focus attentional representation is probably less detailed, as due to the 
distribution of attention, the amount of resources applied to each direction is low. For instance, it may be that focused attention allows for the representation of some distinctions within directions (e.g., between upper left and lower left) that are not available when attention is distributed.

\subsection{Perceptual Character of Content}

The reasoning above shows that empty, egocentric, directional spatial content can be attributed to experiences of pure and partial silence. However, it is still possible that this content is not perceptual, auditory content. First, it may be claimed that the content considered is not perceptual at all. Second, it can be proposed that while it is perceptual, it is not auditory.

There are three ways of denying that this spatial content is not perceptual. According to the first, content is not perceptual but is a higher-order, postperceptual content of a belieflike state. However, this is not likely, as the empirical studies discussed earlier clearly show characteristic behavioral effects—-such as priming - connected with the functioning of attention. Furthermore, there are neuroscientific data that show that resolving such experimental tasks involves neural activation typical for focusing attention [Tata and Ward 2005; $\mathrm{Wu}$ et al. 2007]. In addition, attentional mechanisms are commonly treated as constituting perceptual systems; more specifically, they are considered as constituting 'midlevel' perception [Rensink 2001], and so it is unlikely that content obtained due to the functioning of attention would not be perceptual.

A second option is to postulate that spatial content in experiences of silence is not a product of auditory perceptual processes, but instead arises from operations of auditory imagery. While auditory imagery and perception share some neural mechanisms, obtaining the described priming effects require attending to the actual spatial directions and not merely 
imagining some form of auditory space. In fact, there are data suggesting that attending to the content of imagery interferes with the perceptual processing of stimuli [Villena-González et al. 2016]. Thus, if the spatial content of experiences of silence were merely imagery content, the clear priming effects concerning the processing of external stimuli should not be observed, as focusing on imagined auditory space would limit the attentional resources applied to actual spatial directions. Of course, this does not exclude situations in which auditory imagery serves as a causal factor leading to a perceptual experience, with perceptual content, in which attention is directed to the right or left.

Finally, one may worry that postulating empty spatial content entails that perception can present negative states of affairs, that is, it can present the absence of some entities. However, it is controversial whether such negative states can be perceptually represented [Molnar 2000]. Nevertheless, as observed by Soteriou [2011: 192-3], a perceptual presentation of an empty space should not be interpreted as a case of a negative perception. In particular, the postulated spatial content, such as there is an empty direction 'left,' is distinct from negative content, such as there are no sounds in the 'left' direction. In the first case, perception does not present a negative state, such as a lack of sounds, but presents a positive entity - a spatial direction - and attributes to it a feature of being empty. In the case of fragments of space, being empty is not merely an absence of a property but is a positive feature corresponding to one of the possible states of space, one equally 'positive' as other states concerning, for instance, containing a sound or being filled with a green color.

Another idea is to postulate that while spatial content of experiences of silence is perceptual, it is not auditory. In particular, one may propose that if the direction of attention in the experiments considered is preceded by seeing a right-facing arrow, then what is focused is not auditory but visual attention. In consequence, the relevant spatial content is visual. However, such hypothesis is not particularly plausible, as it is difficult to explain why purely 
visual attention would enhance the processing of an auditory stimulus. Furthermore, there are electrophysiological studies showing that, modality specific, auditory event-related potentials are observed in auditory priming studies [Eimer and Driver 2001; Tata and Ward 2005]. The presence of such event-related potentials suggests the employment of auditory, and not merely visual or some other form of attention. In addition, space presented by vision has a distinct structure from space presented by audition, as spatial directions perpendicular to the right and left ear are positioned outside the visual spatial framework. In consequence, it is unlikely that they can be selected by visual spatial attention.

A possibly more promising idea is to state that the spatial content of experiences of silence is an amodal perceptual content, as it arises from applying attentional resources that are not specific to any modality. However, there are behavioral data suggesting that the attentional resources used in priming studies are not merely amodal but that modality specific auditory resources are employed. It has been observed that if auditory attention is endogenously directed toward the right or left, then not just auditory stimuli, but visual stimuli presented on that side are processed more efficiently. This suggests that some of the attentional resources used are indeed not modality specific but shared - at least between audition and vision. However, in such experiments, attentional effects regarding the processing of auditory stimuli are stronger than those regarding the processing of visual stimuli [Spence and Driver 1996]. In consequence, it seems that while some attentional resources are shared, and so may be amodal, specific auditory attentional resources are also used when people listen, to determine whether there are sounds positioned on the right or on the left.

I do not claim that perceptual content in the case of experiences of silence is purely auditory without any amodal, visual, or other components. Such a strong thesis would be difficult to defend in case of any spatial content, as even vision uses nonvisual information in 
representing space-for instance, provided by proprioception [Alsmith 2017]. However, the above considerations demonstrate an important role of specific auditory resources and so suggest a presence of auditory content.

\subsection{Phenomenality of Content}

Finally, even if we agree that the content considered is perceptual and auditory, whether it is phenomenal may still be in doubt. As I stated in the introduction, by mental states possessing phenomenal content I understand such states that one can introspectively recognize having them. In the previous sections, I have argued that priming studies demonstrate the ability to focus auditory attention towards soundless directions 'left' and 'right'. Furthermore, I have provided reasons for belief that such different foci of attention correspond to experiences of silence with distinct perceptual, spatial contents. In addition, it is plausible to maintain that attention to the soundless direction 'right' has a distinct phenomenal character from focusing to the soundless direction 'left'. This, too, is demonstrated by the priming studies; to succeed in such tasks, one must possess the ability to recognize what is it like to have attention focused toward a particular soundless direction in order to control that a specific action (like focusing to the left and not to the right) is taken. The fact that people participating in priming tasks have no difficulty in recognizing that they have focused to the left or to the right suggests that there is some phenomenal difference between each attentional focus. In consequence, experiences of silence generated by focusing to the left have distinct perceptual spatial contents and distinct phenomenal characters from experiences of silence generated by focusing to the right. Furthermore, because these phenomenal characters change along with representational perceptual contents, is plausible to accept that phenomenal character supervenes on perceptual, spatial content in experiences of silence. 
Nevertheless, despite the plausibility of the above picture, one may propose an alternative model according to which focusing to the left or right generates a propositional state, such as a belief, with certain cognitive phenomenology. According to such a view, while one can introspectively recognize having an experience of silence, recognition happens not due to perceptual phenomenology, but merely in virtue of cognitive phenomenology associated with the formed belief. In other words, while distinct attentional foci correspond to distinct perceptual contents, on a phenomenal level they correspond to distinct cognitive — not perceptual - phenomenal characters. However, I believe that such a cognitive interpretation has a disadvantage that is absent in an interpretation in terms of perceptual phenomenology. Attentional processing of an element $X$ closely correlates with having an experience with a perceptual phenomenal character determined by the attended element $X$ [Prinz 2000; Rensink, 2001]. In fact, cases of attentional processing without modification of perceptual phenomenology happen under specific conditions related to the presentation of brief stimuli or interference between stimuli [Boxtel et al. 2010]. However, such conditions are not satisfied in many experiences of silence, as silence can be prolonged and be perceived without relevant sensory interference. In consequence, a proponent of a thesis that phenomenology of experiences of silence is merely cognitive has to provide an additional explanation of why, in such experiences attention function differently than in other experiences, such that differences in attentionally obtained perceptual contents are not associated with phenomenal, perceptual differences. However, the interpretation in terms of perceptual phenomenology is free from this problem, as it holds that the relation between attention and perceptual phenomenology is not different in experiences of silence than in other types of perceptual experiences.

\section{Temporal Content and Problems of Spatial Account}


In the previous section, I argued that even experiences of pure silence have egocentric, directional spatial content, and furthermore, that this content is both auditory and phenomenal. However, as stated in the introduction, there is also another theory of experiences of silence, which can be plausibly interpreted as postulating that such experiences have auditory and phenomenal content. According to this theory [Phillips 2013; Soteriou 2011], experiences of pure silence have empty temporal content, as they present that there is a period empty of sounds.

I do not aim to offer an argument which would force us to abandon the temporal theory. In fact, there may be reasons that would justify developing a combined, spatiotemporal notion of experiences of silence. However, I aim to demonstrate that my spatial theory has a certain advantage over a pure temporal theory. More specifically, the justification that the postulated content is actually an auditory content is easier in case of spatial theory than in case of temporal theory.

First, the way in which audition presents time does not seem to be different from the way in which vision, and other senses, present time. In particular, the perceptually presented structure of time seems to be the same no matter the modality. Depending on whether one prefers an A- or B-theory of perceptual time [Kriegel 2009], time is experienced as having a linear structure composed of past, present, and future moments, or as composed of moments organized by 'earlier than'/'later than' relations. Thus, it seems likely that temporal perceptual content is, in fact, amodal content.

Second, the physiological mechanisms responsible for time perception do not seem to be modality specific. Quite the contrary; major mechanisms involved in time perception are amodal, such that their output may be used by various perceptual and cognitive systems (see Burr and Morrone [2006] for a review). This additionally suggests that there is no specific auditory temporal content, but that such content is shared among modalities. In particular, 
even if one lost the physiological structures responsible for auditory perception, and so had no auditory experiences at all, he could still possess experiences with the same temporal content, that means experiences presenting sequentially organized temporal moments, as before that loss. In fact, while deaf people make more mistakes in estimating the temporal duration they are certainly able to perceive stimuli as forming a temporal sequence [Kowalska and Szelag 2006].

Justification of the claim that the content of the experience of silence is auditory is easier in the case of the proposed spatial theory. First, audition in representing space in an important respect relies on processes occurring within the auditory system itself [Moore 1991]. Second, in contrast to the case of temporal content, the experiential spatial structure differs between modalities. Audition presents egocentric spatial directions such as 'right' and 'left' without presenting the continuous spatial field, visual space has the form of a coneshaped field [Richardson 2010], and tactile space is determined by the bodily form [Cheng and Haggard 2018]. In particular, while both vision and audition present egocentric directions, the structures of these egocentric representations are distinct. For instance, egocentric directions perpendicular to the left and right ear are positioned outside the visual field but are clearly present within auditory spatial framework. In consequence, it is likely that if one were lacking auditory experiences due to a loss of function in the auditory system, one would not be able to possess experiences with spatial content structured in a way characteristic of audition, as some egocentric directions are included only within auditory space.

While spatial theory has certain advantages over the temporal theory, it still may be the case that there are problems specific to accounts postulating spatial content of experiences of silence. In fact, three arguments against characterizing experiences of pure silence in terms of spatial content have been presented by Meadows [2020: 244-6]. Below, I argue that they do not threaten my version of spatial theory postulating egocentric, directional content. First, 
Meadows claims that the spatial content of experiences of silence is derivative, as spatial silence perception can happen only when it is directly preceded by the perception of an auditory stimulus positioned in the relevant place. However, I presented evidence in Section 2 suggesting that one can auditorily experience empty, egocentric directions in a non-derivative way.

According to Meadows' second argument, experiences of pure silence are unlikely to have spatial content, as the phenomenal character of such experiences is insensitive to changes concerning the spatial characteristics of silence. For instance, we may imagine a situation in which one is positioned in a completely silent room with volume $V$, and the volume of the room extends to volume $2 \mathrm{~V}$. Despite changing the parameters of the silent space, there would be no change in the phenomenology of an experience of silence. Nevertheless, it should be noted that a proponent of a spatial theory of silence perception does not have to assume that every change in spatial parameters of surrounding silence must entail a change in perceptual phenomenology. Even if the content of experiences of silence is spatial, not all spatial properties of silence have to be represented by such an experience.

For illustration, let's consider a visual experience of total darkness. Plausibly, such an experience has some spatial content, as it presents darkness in all directions. There are many possible changes in the spatial characteristics of darkness that do not cause any differences in the phenomenology of the experience of darkness. For instance, the experience of darkness is phenomenally the same no matter whether a wall blocking the light is five or ten meters from the observer. However, this is not problematic, as the spatial content of the experience probably does not contain detailed information about the size of the space filled by darkness. On the other hand, such experience presents darkness as positioned in various directions, and in fact, changes concerning such directional content are clearly reflected in phenomenology. For instance, if at first darkness were present in all directions, and subsequently only in half of 
all directions, the phenomenology of the corresponding visual experience would change significantly.

The analogous point is also true about my theory postulating the egocentric, directional content of experiences of silence. Such content does not specify the size of the surrounding silence, and so it is not surprising that a person cannot, relying on the phenomenology of the experience of silence, distinguish between being in a room with a volume $V$ and a volume $2 V$. However, what is represented are egocentric directions, and changes in the directional characteristics of silence are clearly reflected in phenomenology. In particular, an experience of pure silence in which empty directions 'right' and 'left' are presented is phenomenally distinct from one of partial silence in which there is no presentation of the empty direction 'right,' but only of the empty direction 'left'.

Finally, Meadows claims that experiences of pure silence are not likely to have spatial content, because having such an experience is not phenomenally distinguishable from being newly deaf. However, if a proponent of a spatial theory claims that the postulated spatial content is phenomenal, as indeed happens in my theory, then, contrary to the intuition expressed by Meadows, there should be a phenomenal difference between experiences of silence and cases of deafness.

First, it should be noted that deafness in a heterogeneous phenomenon. There may be kinds of deafness which consist of not having any auditory experiences (e.g., when auditory cortical structures are severely damaged). However, there may be different kinds of deafness in which one has a constant experience as of silence no matter the environmental circumstances (e.g., when cortical attentional systems are intact but auditory receptors lost their sensitivity). An analogous point can be made regarding the relation between seeing darkness and blindness. While some form of blindness may involve an inability to have visual experiences, other forms may involve a constant perception as of darkness. In consequence, a 
proponent of a spatial theory does not have to, and in fact, should not postulate that every episode of deafness is phenomenally distinct from an experience of pure silence, as some form of deafness may simply consist of having experiences like pure silence.

Nevertheless, spatial theories which postulate phenomenal spatial content should still provide a justification for a thesis that experiences of pure silence are phenomenally distinct from situations in which one lacks auditory experiences, which indeed is likely to occur in some forms of deafness. I believe that my theory has resources to provide such justification. In particular, when having an experience of silence, one may manipulate auditory attention by distributing it toward both directions or by focusing it toward the right or left. As argued in the previous section, the use of auditory attention is likely to be associated with some phenomenal character. On the other hand, when one lacks an auditory experience, one cannot deploy auditory attention in any way - in a manner similar to how we cannot deploy visual attention behind our head-and so lack certain phenomenology. In consequence, my theory provides a justification for the presence of a phenomenal difference between experiences of pure silence and situations in which no auditory experience is present.

\section{Conclusions}

I have argued that even experiences of pure silence possess spatial content. More specifically, it is egocentric, directional content, in virtue of which empty directions 'left' and 'right' are presented. The existence of such content is supported by empirical results concerning the functioning of auditory attention. Furthermore, there are good reasons for postulating that the spatial content considered is perceptual and phenomenal. In consequence, a difference between an experience of silence and the lack of an auditory experience is analogous to a difference between an experience of darkness and the lack of a visual experience- - both the 
experience of silence and of darkness has spatial, perceptual, and phenomenal content. The proposed spatial theory has an advantage over a temporal theory of silence, as it is more plausible that the proposed spatial content is auditory. In addition, the spatial theory formed in terms of egocentric, directional content is not threatened by the usual arguments formulated against spatial theories of silence perception.

\section{Acknowledgements}

The author would like to thank the anonymous reviewers for their comments. The paper was presented during SemDok seminar at Jagiellonian University and 94th Joint Session of the Mind Association and Aristotelian Society. The early idea of the paper was presented when I was a commentator on Phillip Meadows' paper during Auditory Perception and Musical Sound workshop organized by Elvira Di Bona and Michał Klincewicz.

\section{REFERENCES}

Alsmith, A.J.T. 2017. Perspectival Structure And Agentive Self-Location, in The Subject's Matter: Self-consciousness and the Body, eds. Frederique De Vignemont and Adrian Alsmith, Cambridge, MA: MIT Press: 263-88.

van Boxtel, J.J.A., N. Tsuchiya, and C. Koch 2010. Consciousness and Attention: On Sufficiency And Necessity. Frontiers in Psychology 1/217, doi:10.3389/fpsyg.2010.00217.

Burr, D., and C. Morrone 2006. Time Perception: Space-Time in the Brain, Current Biology 16/5: R171-3.

Carrasco, M., and A. Barbot 2019. Spatial Attention Alters Visual Appearance, Current Opinion in Psychology 29: 56-64. 
Cavedon-Taylor, D. 2017. Touching Voids: On the Varieties of Absence Perception, Review of Philosophy and Psychology 8/2: 355-66.

Cheng, T., and P. Haggard 2018. The Recurrent Model of Bodily Spatial Phenomenology, Journal of Consciousness Studies 25/3-4: 55-70.

Eimer, M., and J. Diver 2001. Crossmodal Links in Endogenous and Exogenous Spatial Attention: Evidence from Event-Related Brain Potential Studies, Neuroscience and Biobehavavioral Reviews 25/6: 497-511.

Eimer, M., J. van Velzen, and J. Driver 2004. ERP Evidence for Cross-Modal Audiovisual Effects of Endogenous Spatial Attention within Hemifields, Journal of Cognitive Neuroscience 16/2: 272-88.

Farennikova, A. 2013. Seeing Absence, Philosophical Studies 166/3: 429-54.

Kowalska, J., and E. Szelag 2006. The Effect of Congenital Deafness on Duration Judgment, The Journal of Child Psychology and Psychiatry 47/9: 946-53.

Kriegel, U. 2009. Temporally Token-Reflexive Experiences, Canadian Journal of Philosophy 39/4: 585-617.

Mac Cumhaill, C. 2015. Perceiving Immaterial Paths, Philosophy and Phenomenological Research 90/3: 687-715.

Martin, J.R., and J. Dokic 2013. Seeing Absence or Absence of Seeing?, Thought 2/2: 11725.

Mayer, A.R., D. Harrington, J.C. Adair, and R. Lee 2006. The Neural Networks Underlying Endogenous Auditory Covert Orienting and Reorienting, Neuroimage 30/3: 938-49.

Meadows, P.J. 2020. Experiencing Silence, Canadian Journal of Philosophy 50/2: 238-50.

Molnar, G. 2000. Truthmakers for Negative Truths, Australasian Journal of Philosophy 78/1: $72-86$. 
Mondor, T.A., and M.P. Bryden1992. Orienting of Auditory Spatial Attention: Effects of a Lateralized Tone Cue, Neuropsychologia 30/8: 743-5.

Moore, D.R. 1991. Anatomy and Physiology of Binaural Hearing, Audiology 30/3: 125-34.

Nudds, M. 2009. Sounds and Space, in Sounds and Perception. New Philosophical Essays, eds. Matthew Nudds and Casey O’Callaghan, Oxford: Oxford University Press: 69-96.

O’Callaghan, C. 2008. Object Perception: Vision and Audition, Philosophy Compass 3/4: 803-29.

O’Shaughnessy, B. 2000. Consciousness and The World, Oxford: Oxford University Press.

O'Shaughnessy, B. 2009. The Location of a Perceived Sound, in Sounds and Perception. New Philosophical Essays, eds. Matthew Nudds and Casey O'Callaghan, Oxford: Oxford University Press: 111-25.

Phillips, I. 2013. Hearing and Hallucinating Silence, in Hallucination. Philosophy and Psychology, eds. Fiona Macpherson and Dimitris Platchias, Cambridge, MA: The MIT Press: $333-59$.

Prinz, J. 2000. A Neurofunctional Theory of Visual Consciousness, Consciousness and Cognition 9/2: 243-59.

Rensink, R.A. 2000. The Dynamic Representation of Scenes, Visual Cognition 7/1: 17-42.

Rensink, R.A. 2001. Change Blindness: Implications for the Nature of Visual Attention, in Vision and Attention, eds. Michael Jenkin and Laurence Harris, New York: Springer: 169-88. Richardson, L. 2010. Seeing Empty Space, European Journal of Philosophy 18/2: 227-43. Sach, A.J., N.I. Hill, and P.J. Bailey 2000. Auditory Spatial Attention Using Interaural Time Differences, Journal of Experimental Psychology: Human Perception and Performance 26/2: 717-29.

Schellenberg, S. 2016. Perceptual Particularity, Philosophy and Phenomenological Research 93/1: 25-54. 
Scholl, B. J. 2001. Objects and Attention: The State of Art, Cognition 80/1-2: 1-46.

Schröger, E., and M. Eimer. 1997. Endogenous Covert Spatial Orienting in Audition: "CostBenefit" Analyses of Reaction Times and Event-Related Potentials, The Quarterly Journal of Experimental Psychology A: Human Experimental Psychology 50A/2: 457-74.

Siegel, S. 2010. Contents of Visual Experience, New York: Oxford University Press.

Sorensen, R. 2004. We See in the Dark, Nô̂s 38/3: 456-80.

Sorensen, R. 2009. Hearing Silence: The Perception and Introspection of Absences, in Sounds and Perception. New Philosophical Essays, eds. Matthew Nudds and Casey O'Callaghan, Oxford: Oxford University Press: 126-45.

Soteriou, M. 2011. The Perception of Absence, Space, and Time, in Perception, Causation, and Objectivity, eds. Johannes Roessler, Hemdat Lerman and Naomi Eilan, Oxford: Oxford University Press: 181-206.

Spence, C., and J. Driver (1996). Audiovisual Links in Endogenous Covert Spatial Attention, Journal of Experimental Psychology: Human Perception and Performance 22/4, 1005-30.

Teder-Sälejärvi, W.A., and S.A. Hillyard 1998. The Gradient of Spatial Auditory Attention in Free Field: An Event-Related Potential Study, Perception and Psychophysics 60/7: 1228-42.

Villena-González, M., V. López, and E. Rodríguez. 2016. Orienting Attention to Visual or Verbal/Auditory Imagery Differentially Impairs the Processing of Visual Stimuli, NeuroImage 132/15: 71-8.

Voisin, J., A. Bidet-Caulet, O. Bertrand, and P. Fonlupt. 2006. Listening in Silence Activates Auditory Areas: A Functional Magnetic Resonance Imaging Study, Journal of Neuroscience 26/1: 273-8.

Wright, R. 2012. Darkness visible?, Australasian Journal of Philosophy 90/1: 39-55. 
Wu, C.-T., D.H. Weissman, K.C. Roberts, and M.G. Woldorff. 2007. The Neural Circuitry Underlying the Executive Control of Auditory Spatial Attention, Brain Research 1134/1: 187 98.

Tata, M.S., and L.M. Ward. 2005. Spatial Attention Modulates Activity in a Posterior “Where” Auditory Pathway. Neuropsychologia 43/4: 509-16.

Treisman, A.M. 1998. Feature Binding, Attention and Object Perception. Philosophical Transactions of the Royal Society of London. Series B. Biological sciences 353/1373: 1295306.

Young, N. 2017. Hearing Spaces. Australasian Journal of Philosophy 95/2: 242-55.

\section{Funding Information}

The work was supported by the National Science Center (Poland) grant 2018/31/D/HS1/00363.

\section{Institutional Affiliation}

Institute of Philosophy, Jagiellonian University, Kraków, Poland 